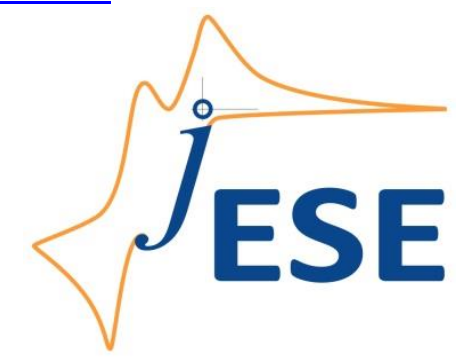

Open Access : : ISSN 1847-9286

www.jESE-online.org

Original scientific paper

\title{
Simulation of square wave voltammetry of three electrode reactions coupled by two reversible chemical reactions
}

\author{
Milivoj Lovrić \\ Divkovićeva 13, Zagreb 10090, Croatia \\ mlovric@irb.hr
}

Received: July 15, 2017; Accepted: August 7, 2017

\begin{abstract}
Three fast and reversible electrode reactions that are connected by two reversible chemical reactions that are permanently in the equilibrium are analysed theoretically for square wave voltammetry. The dependence of peak potentials on the dimensionless equilibrium constants of chemical reactions is calculated. The influence of the basic thermodynamic parameters on the square wave voltammetric responses is analysed.
\end{abstract}

\section{Keywords}

ECECE mechanism; equilibrium conditions; voltammetry; theory

\section{Introduction}

Multistep electrode processes appear in electrochemistry of many organic compounds [1-9]. Some of these mechanisms include chemical steps [10-15]. In this communication, a theory of three electrode reactions that are coupled by two reversible chemical reactions is developed for square wave voltammetry under equilibrium conditions. The acronym of this mechanism is ECECE $[16,17]$. It was observed in reductions of dibromonitrobenzene [17] and benzoquinone dioxime [18-20] and oxidation of catechol in methanol [21,22]. Theoretical models of ECECE are developed for reversible electrode reactions and totally irreversible chemical reactions in cyclic voltammetry [16] and chronoamperometry $[17,19]$.

\section{The model}

It is assumed that the product of the first electrode reaction is the reactant of the first chemical reaction and that the product of the latter is the reactant of the second electrode reaction. Also, it is assumed that the reactant and the product of the second chemical reaction connect the second and the third electrode reactions in the same way:

$$
A \leftrightarrow B^{+}+e^{-}
$$




$$
\begin{aligned}
& \mathrm{B}^{+}+\mathrm{X}^{-} \leftrightarrow \mathrm{E} \\
& \mathrm{E} \leftrightarrow \mathrm{F}^{+}+\mathrm{e}^{-} \\
& \mathrm{F}^{+}+\mathrm{Y}^{-} \leftrightarrow \mathrm{G} \\
& \mathrm{G} \leftrightarrow \mathrm{H}^{+}+\mathrm{e}^{-}
\end{aligned}
$$

Furthermore, it is assumed that the mass transport can be described by the stationary, planar, semiinfinite diffusion model and that the reagents $X^{-}$and $Y^{-}$are present in great excess, so that the variation of their concentrations can be neglected. Finally, it is assumed that both chemical reactions are permanently in the equilibrium. The model is based on the following differential equations:

$$
\begin{aligned}
& \partial c_{\mathrm{A}} / \partial t=D \partial^{2} c_{\mathrm{A}} / \partial x^{2} \\
& \partial c_{\text {Int }_{1}} / \partial t=D \partial^{2} c_{\text {Int }_{1}} / \partial x^{2} \\
& \partial c_{\text {Int }_{2}} / \partial t=D \partial^{2} c_{\text {Int }_{2}} / \partial x^{2} \\
& \partial c_{\mathrm{H}} / \partial t=D \partial^{2} c_{\mathrm{H}} / \partial x^{2} \\
& c_{\text {Int }_{1}}=c_{\mathrm{B}}+c_{\mathrm{E}} \\
& c_{\text {Int }_{2}}=c_{\mathrm{F}}+c_{\mathrm{G}} \\
& t=0, \quad x \geq 0: \quad c_{\mathrm{A}}=c_{\mathrm{A}}^{*}, c_{\mathrm{B}}=c_{\mathrm{E}}=c_{\mathrm{F}}=c_{\mathrm{G}}=c_{\mathrm{H}}=0 \\
& c_{\mathrm{X}}=c_{\mathrm{X}}^{*}, c_{\mathrm{Y}}=c_{\mathrm{Y}}^{*} \\
& t>0, x \rightarrow \infty: \quad c_{A} \rightarrow c_{\mathrm{A}}^{*}, c_{\mathrm{X}} \rightarrow c_{\mathrm{X}}^{*}, c_{\mathrm{Y}} \rightarrow c_{\mathrm{Y}}^{*}, c_{\mathrm{B}} \rightarrow 0 \\
& c_{\mathrm{E}} \rightarrow 0, c_{\mathrm{F}} \rightarrow 0, c_{\mathrm{G}} \rightarrow 0, c_{\mathrm{H}} \rightarrow 0 \\
& x=0: \quad c_{\mathrm{X}}=c_{\mathrm{X}}^{*}, \quad c_{\mathrm{Y}}=c_{\mathrm{Y}}^{*} \\
& c_{\mathrm{B}, \mathrm{X}=0}=c_{\mathrm{A}, \mathrm{x}=0} \exp \left(F\left(E-E_{1}^{0}\right) / R T\right) \\
& K_{1} c_{X}^{*}=\mathrm{c}_{\mathrm{E}, \mathrm{x}=0} / \mathrm{c}_{\mathrm{B}, \mathrm{X}=0} \\
& c_{\mathrm{F}, \mathrm{X}=0}=c_{\mathrm{E}, \mathrm{X}=0} \exp \left(F\left(E-E_{2}^{0}\right) / R T\right) \\
& K_{2} c_{\mathrm{Y}}^{*}=c_{\mathrm{G}, \mathrm{x}=0} / c_{\mathrm{F}, \mathrm{x}=0} \\
& c_{\mathrm{H}, \mathrm{x}=0}=c_{\mathrm{G}, \mathrm{X}=0} \exp \left(F\left(E-E_{3}^{0}\right) / R T\right) \\
& D\left(\partial c_{\mathrm{A}} / \partial x\right)_{\mathrm{x}=0}=I_{1} / F S \\
& D\left(\partial c_{\text {Int }_{1}} / \partial x\right)_{\mathrm{x}=0}=\left(I_{2}-I_{1}\right) / F S \\
& D\left(\partial c_{\text {Int }_{2}} / \partial x\right)_{\mathrm{x}=0}=\left(I_{3}-I_{2}\right) / F S \\
& D\left(\partial c_{\mathrm{H}} / \partial x\right)_{\mathrm{x}=0}=-I_{3} / F S \\
& x>0: K_{1} c_{\mathrm{X}}^{*}=c_{\mathrm{E}} / c_{\mathrm{B}} \\
& K_{2} c_{\mathrm{Y}}^{*}=c_{\mathrm{G}} / c_{\mathrm{F}}
\end{aligned}
$$

The meanings of symbols are reported in Table 1 . Differential equations are solved by the numerical method [23]. The solution is the system of recursive formulae for the dimensionless current:

$$
\Phi_{2, m}=u_{5}\left(u_{1}+u_{2}-u_{3}-u_{4}\right)^{-1}-\sum_{\mathrm{j}=1}^{\mathrm{m}-1} \Phi_{2, \mathrm{j}} S_{\mathrm{m}-\mathrm{j}+1}
$$


Table 1. Meanings of symbols

\begin{tabular}{ll}
\hline$c_{A}, c_{B}, c_{E}, c_{F}, c_{G}, c_{H}$ & Concentrations of species $\mathrm{A}, \mathrm{B}^{+}, \mathrm{E}, \mathrm{F}^{+}, \mathrm{G}$ and $\mathrm{H}^{+}$ \\
\hline$c_{A}^{*}, c_{X}^{*}, c_{Y}^{*}$ & Concentrations of species $\mathrm{A}, \mathrm{X}^{-}$and $\mathrm{Y}^{-}$in the bulk of solution \\
\hline$D$ & Common diffusion coefficient \\
\hline$E$ & Electrode potential \\
\hline$E_{1}^{0}, E_{2}^{0}, E_{3}^{0}$ & Standard potentials of the first, the second and the third electrode reactions \\
\hline$d E$ & Potential step in square wave voltammetry \\
\hline$E_{S W}$ & Square wave amplitude \\
\hline$E_{p}$ & Peak potential \\
\hline$F$ & Faraday constant \\
\hline$f$ & Square wave frequency \\
\hline$I_{1}, I_{2}, I_{3}$ & Currents of the first, the second and the third electron transfers \\
\hline$R$ & Equilibrium constants of the first and the second chemical reactions \\
\hline$S$ & Gass constant \\
\hline$T$ & Electrode surface area \\
\hline
\end{tabular}

$\Phi_{1, \mathrm{~m}}=u_{6}+u_{7} \sum_{\mathrm{j}=1}^{\mathrm{m}} \Phi_{2, \mathrm{j}} S_{\mathrm{m}-\mathrm{j}+1}-\sum_{\mathrm{j}=1}^{\mathrm{m}-1} \Phi_{1, \mathrm{j}} S_{\mathrm{m}-\mathrm{j}+1}$

$\Phi_{3, m}=u_{8} \sum_{\mathrm{j}=1}^{\mathrm{m}} \Phi_{2, \mathrm{j}} S_{\mathrm{m}-\mathrm{j}+1}-\sum_{\mathrm{j}=1}^{\mathrm{m}-1} \Phi_{3, \mathrm{j}} S_{\mathrm{m}-\mathrm{j}+1}$

$\Phi_{\mathrm{k}}=I_{\mathrm{k}}\left(F S c_{\mathrm{A}}^{*}\right)^{-1}(D f)^{-1 / 2}$

$s_{\mathrm{p}}=\sqrt{p}-\sqrt{p-1}$

$\mathrm{m}=1,2,3 \ldots$.

$u_{1}=\left(1+K_{2} c_{\mathrm{Y}}^{*}\right)^{-1}$

$u_{2}=K_{1} c_{\mathrm{X}}^{*}\left(1+K_{1} c_{\mathrm{X}}^{*}\right)^{-1} \exp \left(F\left(E-E_{2}^{0}\right) / R T\right)$

$u_{3}=u_{2}\left[1+\left(1+K_{1} c_{\mathrm{X}}^{*}\right) \exp \left(F\left(E-E_{1}^{0}\right) / R T\right)\right]^{-1}$

$u_{4}=u_{1} z_{3}\left(1+z_{3}\right)^{-1}$

$z_{3}=K_{2} c_{\mathrm{Y}}^{*} u_{1} \exp \left(F\left(E-E_{3}^{0}\right) / R T\right)$

$u_{5}=5 \sqrt{\pi / 2} u_{2} z_{1}\left(1+z_{1}\right)^{-1}$

$z_{1}=\left(1+K_{1} c_{\mathrm{X}}^{*}\right) \exp \left(F\left(E-E_{1}^{0}\right) / R T\right)$

$u_{6}=5 \sqrt{\pi / 2} z_{1}\left(1+z_{1}\right)^{-1}$

$u_{7}=\left(1+z_{1}\right)^{-1}$

$u_{8}=z_{3}\left(1+z_{3}\right)^{-1}$

The sum $\Phi=\Phi_{1}+\Phi_{2}+\Phi_{3}$ is reported as a function of electrode potential.

\section{Results and discussion}

Square wave voltammograms of the mechanism (1) - (5) under equilibrium conditions depend on the standard potentials of electron transfers and the dimensionless constants $K_{1} c_{X}^{*}$ and $K_{2} c_{Y}^{*}$ of chemical reactions. An ideal case in which the concentrations of reagents $X^{-}$and $Y^{-}$can be changed experimentally from zero to the limit of solubility is investigated here. If $E_{1}^{0}=E_{2}^{0}=E_{3}^{0}, K_{1} c_{\mathrm{X}}^{*}=1$ and $K_{2} C_{\mathrm{Y}}^{*}=1$, the response is a single peak with the dimensionless net peak current $\Delta \Phi_{\mathrm{p}}=2.44$ that 
appears at $E_{\mathrm{p}}=E_{1}^{0}$. This is shown in Fig. 1 . The real peak current depends linearly on the square root of frequency, but the net peak potential is independent of frequency. If the concentration of $Y^{-}$ is reduced to zero, the third electrode reaction vanishes and the net peak current is diminished to 1.50 but the peak potential does not change. Finally, if the concentrations of both $\mathrm{X}^{-}$and $\mathrm{Y}^{-}$are set to zero, only the first electrode reaction occurs and the net peak current is 0.75 at the same peak potential.

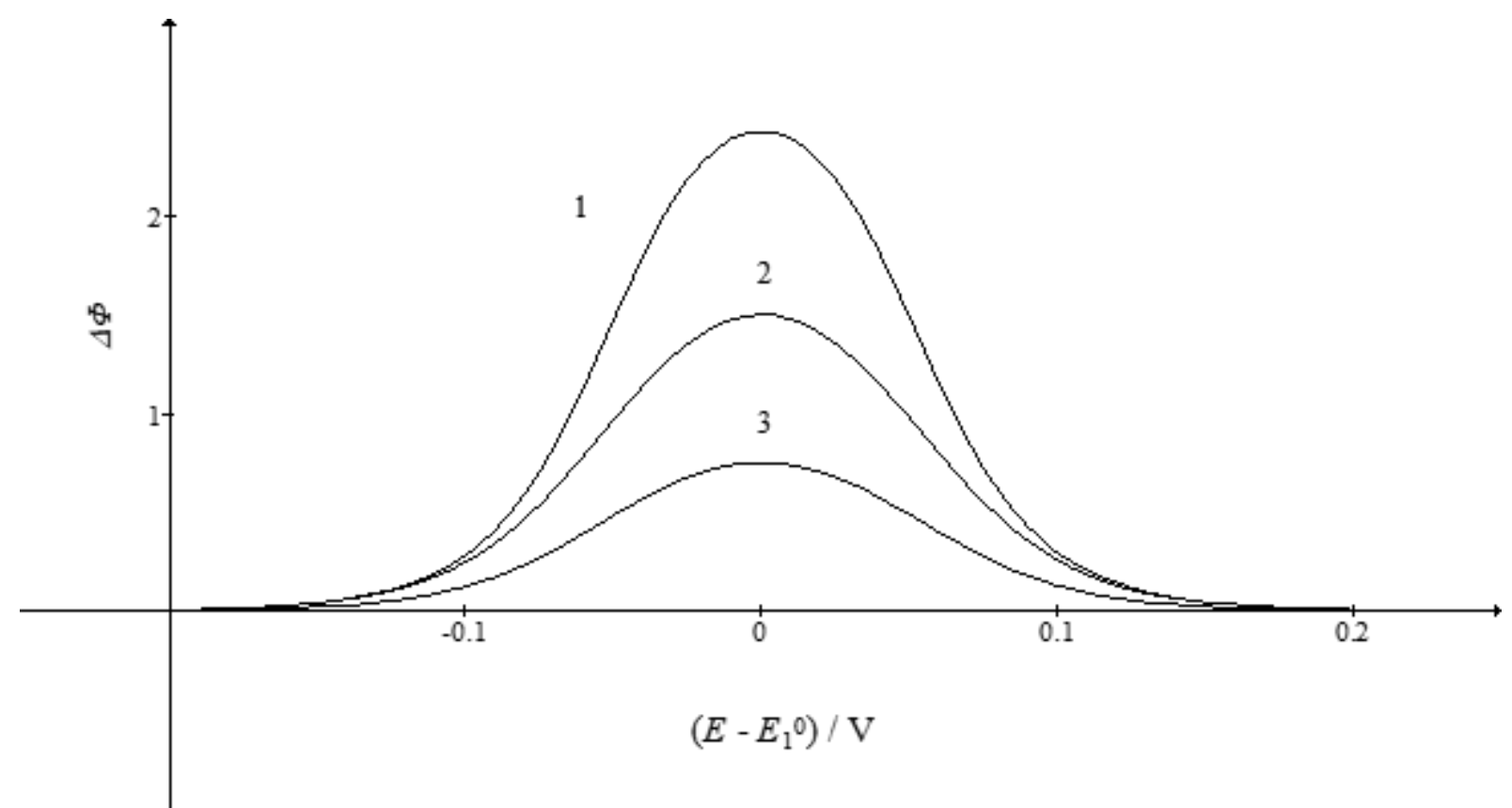

Fig. 1 Dimensionless square wave voltammograms of electrode reactions (1) - (5).

$E_{1}^{0}=E_{2}^{0}=E_{3}^{0}, d E=5 \mathrm{mV}$ and $E_{S W}=50 \mathrm{mV} . K_{1} c_{X}^{*}=1$ and $K_{2} c_{Y}^{*}=1(1), K_{1} c_{X}^{*}=1$ and $K_{2} c_{Y}^{*}=0$ (2) and $K_{1} c_{X}^{*}=0$ and $K_{2} c_{Y}^{*}=0$ (3)

Fig. $2 \mathrm{~A}$ shows that the variation of $\mathrm{X}^{-}$concentration may lead to the split response. If $K_{1} c_{\mathrm{X}}^{*}=300$ and $K_{2} c_{\mathrm{Y}}^{*}=1$, two peaks appear at $-0.135 \mathrm{~V}$ and $0 \mathrm{~V}$ vs. $E_{1}^{0}$. The peak currents are 0.81 and 1.53 , respectively, which means that the first electron is transferred at lower potential than the other two. If $K_{1} c_{\mathrm{X}}^{*}=1000$ the first peak potential is $-0.175 \mathrm{~V}$ vs. $E_{1}^{0}$ and if $K_{1} c_{\mathrm{X}}^{*}=100$ only a shoulder appears at about $-0.13 \mathrm{~V}$, both for $K_{2} c_{\mathrm{Y}}^{*}=1$. These indicate that the first chemical reaction consumes the first product $\mathrm{B}^{+}$and decreases the formal potential of the first electrode reaction. The influence of $\mathrm{Y}^{-}$concentration is shown in Fig. 2B. If $K_{2} c_{\mathrm{Y}}^{*}=300$ the peak currents are 1.71 and 0.78 and the peak potentials are $-0.145 \mathrm{~V}$ and $-0.005 \mathrm{~V}$ vs. $E_{1}^{0}$. As the second chemical reaction consumes the product of the second electrode reaction, the formal potentials of both the first and the second electron transfers are diminished and only the third transfer occurs at the standard potential. These explanations are confirmed by the relationships between net peak potentials and the logarithm of dimensionless constants $K_{1} c_{\mathrm{X}}^{*}$ and $K_{2} c_{\mathrm{Y}}^{*}$ that are shown in Fig. 3. If $K_{2} c_{\mathrm{Y}}^{*}=1$ and $K_{1} c_{\mathrm{X}}^{*}>300$ the potential of the first peak is given by the formula: $E_{\mathrm{p}, 1}-E_{1}^{0}=-2.3(R T / F) \log \left(K_{1} c_{\mathrm{X}}^{*}\right)+0.005 \mathrm{~V}$, which is predicted for an EC mechanism in the equilibrium [24]. The slope of the line 1 in Fig. 3A is $0.020 \mathrm{~V}$. It corresponds to the single peak responses. This slope can be explained by the theory of EEE mechanism with unstable intermediates [3]:

$$
\begin{aligned}
& E_{\mathrm{p}}=\left[E_{1}^{0}-(R T / F) \ln \left(K_{1} c_{\mathrm{X}}^{*}\right)+E_{2}^{0}-(R T / F) \ln \left(K_{2} c_{\mathrm{Y}}^{*}\right)+E_{3}^{0}\right] / 3 \\
& E_{\mathrm{p}}=\left(E_{1}^{0}+E_{2}^{0}+E_{3}^{0}\right) / 3-2.3(R T / 3 F) \log \left(K_{1} c_{\mathrm{X}}^{*}\right)
\end{aligned}
$$



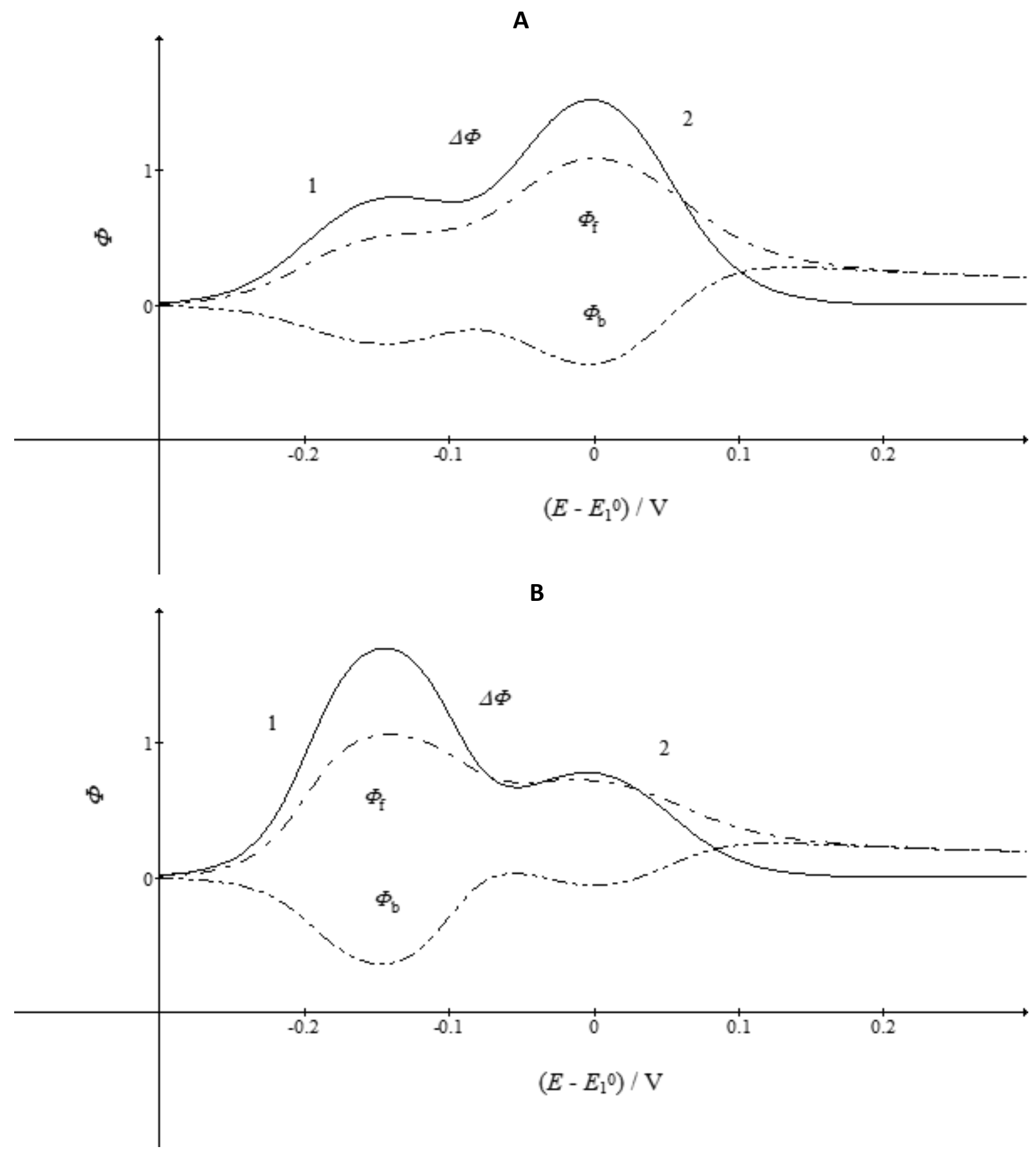

Fig. 2. Square wave voltammograms of electrode reactions (1) - (5). Net responses and their forward and backward components are shown. $E_{1}^{0}=E_{2}^{0}=E_{3}^{0}, K_{1} c_{X}^{*}=300$ and $K_{2} c_{Y}^{*}=1(A)$ and 300 (B). All other parameters are as in Fig. 1

If $30<K_{1} c_{\mathrm{X}}^{*}<300$ the main peak is preceded by the shoulder at about $-0.13 \mathrm{~V}$. The variation of the second dimensionless constant is shown in Fig. 3B. The potential of the single peak is defined by eq. (42) if $-1<\log \left(K_{2} c_{Y}^{*}\right)<2$. Outside this range either the responses with shoulders or the split responses appear. If $K_{2} c_{\mathrm{Y}}^{*}<0.005$ the concentration of the third reactant $\mathrm{G}$ is much smaller than the concentration of the second product $\mathrm{F}^{+}$and the formal potential of the third electrode reaction is higher than the third standard potential.

At this point several special cases can be analyzed. The first one is the ECE mechanism that appears if $K_{2} C_{\mathrm{Y}}^{*}=0$. Fig. $4 \mathrm{~A}$ shows the relationship between peak potentials and the logarithm of the first dimensionless constant. The slopes of straight lines 1,2 and 3 are $-0.03,-0.06$ and $-0.06 \mathrm{~V}$, respectively. 

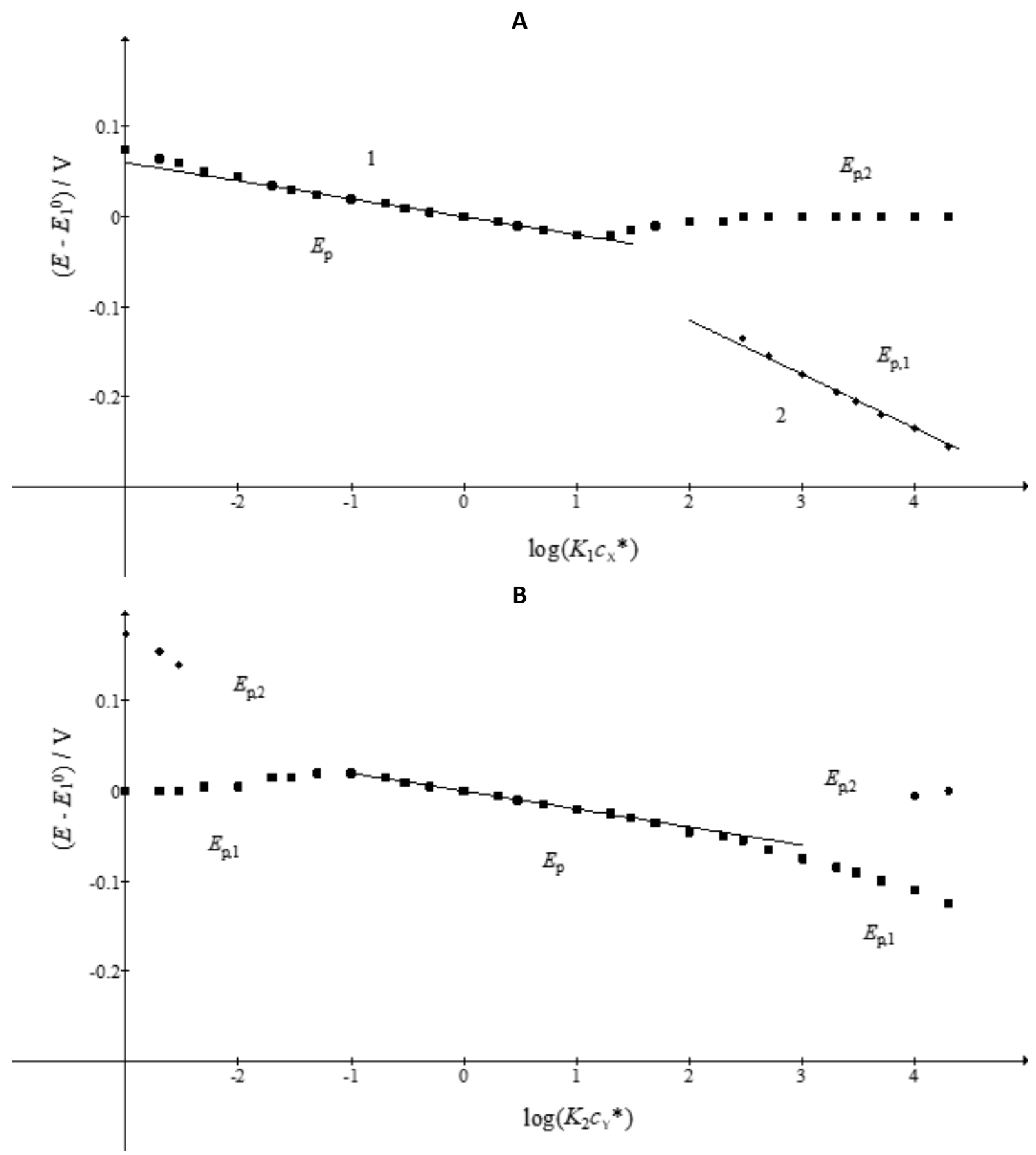

Fig. 3. Dependence of net peak potentials on the logarithm of dimensionless equilibrium constants of chemical reactions (2) and (4). $E_{1}^{0}=E_{2}^{0}=E_{3}^{0}$ and $K_{2} c_{Y}^{*}=1$ (A) and $K_{1} c_{X}^{*}=1$ (B).

The lines are linear approximations

The first slope corresponds to the average of the formal potentials of the first and the second electrode reaction:

$$
\begin{aligned}
& E_{\mathrm{p}}=\left[E_{1}^{0}-(R T / F) \ln \left(K_{1} c_{\mathrm{X}}^{*}\right)+E_{2}^{0}\right] / 2 \\
& E_{\mathrm{p}}=\left(E_{1}^{0}+E_{2}^{0}\right) / 2-2.3(R T / 2 F) \log \left(K_{1} c_{\mathrm{X}}^{*}\right)
\end{aligned}
$$

The slopes of the second and the third straight lines correspond to either EC, or CE mechanism in the equilibrium [24]. This agrees with the previous calculations [15]. Very similar relationships appear in the ECECE mechanism in which the standard potential of the third electrode reaction is much higher than the other two standard potentials. This can be seen in Fig. 4B. The slopes of straight lines in Figs $4 \mathrm{~A}$ and $4 \mathrm{~B}$ are identical. 

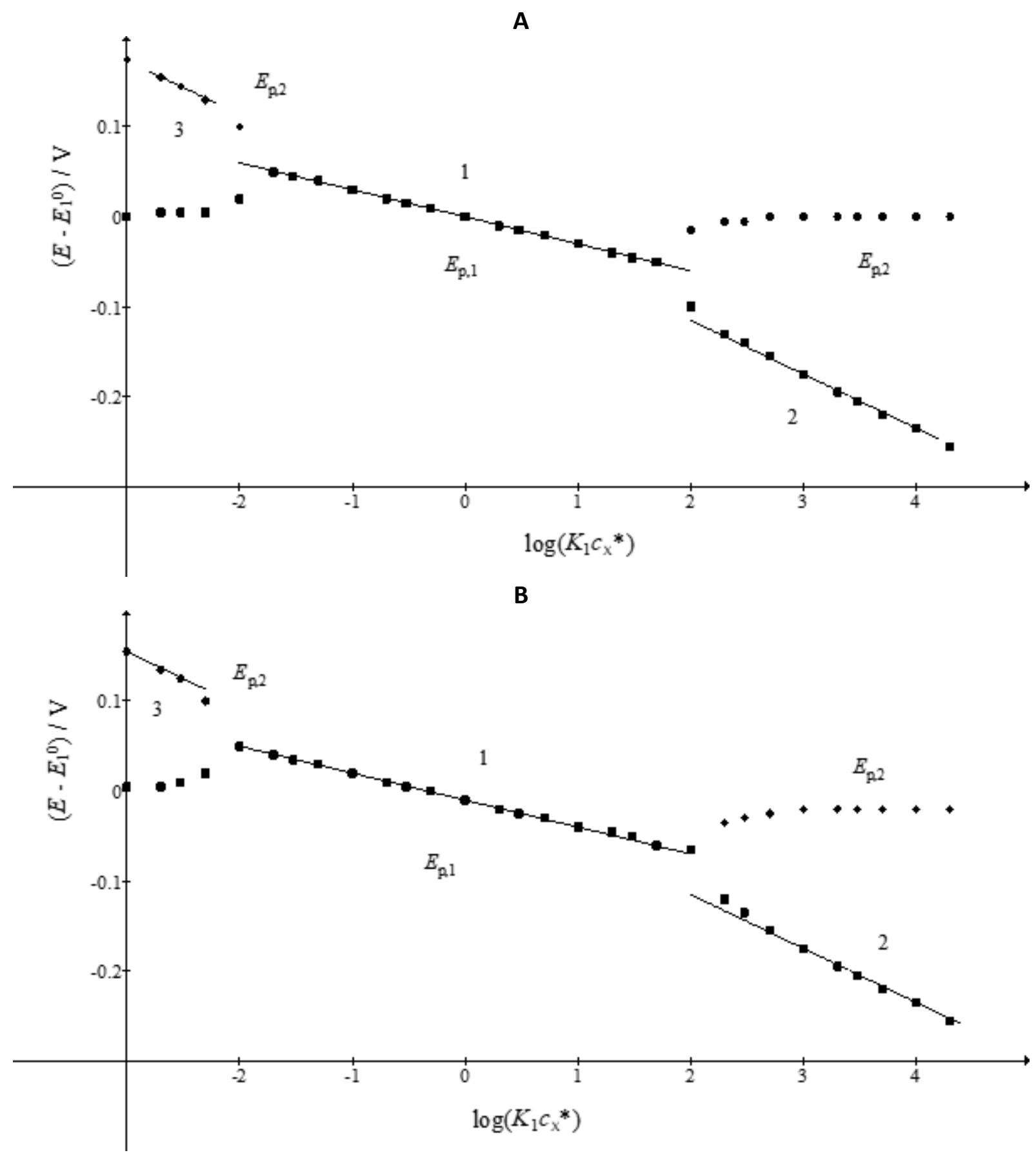

Fig. 4. Dependence of net peak potentials on the logarithm of dimensionless equilibrium constant of the first chemical reaction. $E_{1}^{0}=E_{2}^{0}=E_{3}^{0}$ and $K_{2} c_{Y}^{*}=O(A)$ and $E_{1}^{0}=E_{2}^{0}, E_{3}^{0}-E_{1}^{0}=0.5 V$ and $K_{2} c_{Y}^{*}=1(B)$. The lines are linear approximations

The second special case is the influence of the reagent $Y^{-}$on the second and the third electrode reactions that are separated from the first electrode reaction. If the first standard potential is $400 \mathrm{mV}$ lower than the other two standard potentials and if $K_{1} c_{\mathrm{X}}^{*}=1$, the response consists of either two, or three peaks. This is shown in Fig. 5A. The first peak is independent of $K_{2} C_{\mathrm{Y}}^{*}$ while the dependence of the second and the third peaks on the logarithm of $K_{2} c_{Y}^{*}$ resembles the relationships of the ECE mechanism. Within a wide range $-2<\log \left(K_{2} C_{\mathrm{Y}}^{*}\right)<2$ the second peak satisfies the straight line $E_{\mathrm{p}, 2}-E_{1}^{0}=-0.03 \log \left(K_{2} c_{\mathrm{Y}}^{*}\right)+0.410 \mathrm{~V}$. If $K_{2} c_{\mathrm{Y}}^{*}>300$ the second peak is defined by the equation $E_{\mathrm{p}, 2}-E_{1}^{0}=-0.06 \log \left(K_{2} c_{\mathrm{Y}}^{*}\right)+0.425 \mathrm{~V}$ and the third peak potential is equal to the third standard potential. If $K_{2} c_{Y}^{*}<0.001$ the second peak tends to $0.42 \mathrm{~V}$ and the third peak approaches the asymptote $E_{\mathrm{p}, 3}-E_{1}^{0}=-0.06 \log \left(K_{2} c_{\mathrm{Y}}^{*}\right)+0.395 \mathrm{~V}$. However, if the separation of the first electrode 
reaction from the other two is not high enough, the responses of the first and the second reactions may overlap under certain conditions. Fig. 5B shows the relationships between peak potentials and the logarithm of $K_{2} C_{\mathrm{Y}}^{*}$ for the differences $E_{2}^{0}-E_{1}^{0}=0.2 \mathrm{~V}$ and $E_{3}^{0}-E_{1}^{0}=0.2 \mathrm{~V}$. This figure differs from Fig. 5A if $K_{2} C_{\mathrm{Y}}^{*}>100$. Under the influence of high value of the second dimensionless constant, the formal potential of the second electrode reaction is diminished and approaches the first standard potential. The line 2 in Fig. $5 \mathrm{~B}$ is defined by the equation: $E_{\mathrm{p}, 1}-E_{1}^{0}=-0.03 \log \left(K_{2} c_{\mathrm{Y}}^{*}\right)+$ $+0.1 \mathrm{~V}$. This means that it satisfies the following relationship:

$$
E_{\mathrm{p}}=\left(E_{1}^{0}+E_{2}^{0}\right) / 2-2.3(R T / 2 F) \log \left(K_{2} c_{\mathrm{Y}}^{*}\right)
$$
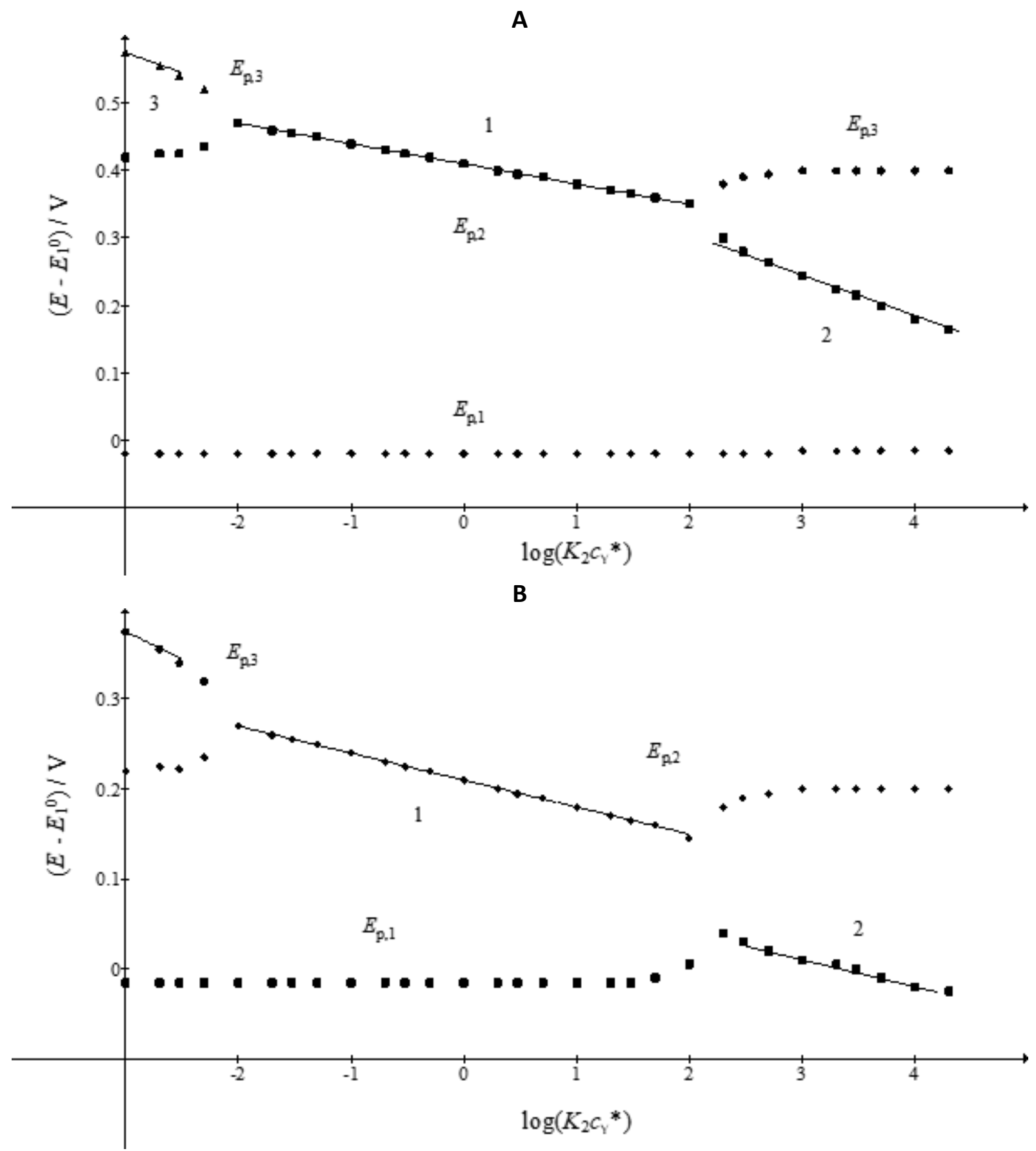

Fig. 5. Dependence of net peak potentials on the logarithm of dimensionless equilibrium constant of the second chemical reaction. $K_{1} c_{X}^{*}=1$ and $E_{2}^{0}-E_{1}^{0}=0.4 \mathrm{~V}$ and $E_{3}^{0}-E_{1}^{0}=0.4 \mathrm{~V}(\mathrm{~A})$ and $E_{2}^{0}-E_{1}^{0}=0.2 \mathrm{~V}$ and $E_{3}^{0}-E_{1}^{0}=0.2 \vee(B)$ 
These mixed responses can be formed only if the difference between formal potentials is smaller than $0.1 \mathrm{~V}$ [3]. If this condition is not satisfied, three peaks appear at all concentrations of the reagent $Y^{-}$. An example is shown in Fig. 6 . The straight lines 1 and 2 are given by the equations: $E_{\mathrm{p}, 3}-E_{1}^{0}=-0.06 \log \left(K_{2} c_{\mathrm{Y}}^{*}\right)+0.4 \mathrm{~V}$ and $E_{\mathrm{p}, 2}-E_{1}^{0}=-0.06 \log \left(K_{2} c_{\mathrm{Y}}^{*}\right)+0.32 \mathrm{~V}$, respectively. They demonstrate that the second chemical reaction influences only the second electrode reaction if $K_{2} c_{\mathrm{Y}}^{*}>10$ and only the third electrode reaction if $K_{2} c_{\mathrm{Y}}^{*}<0.1$.

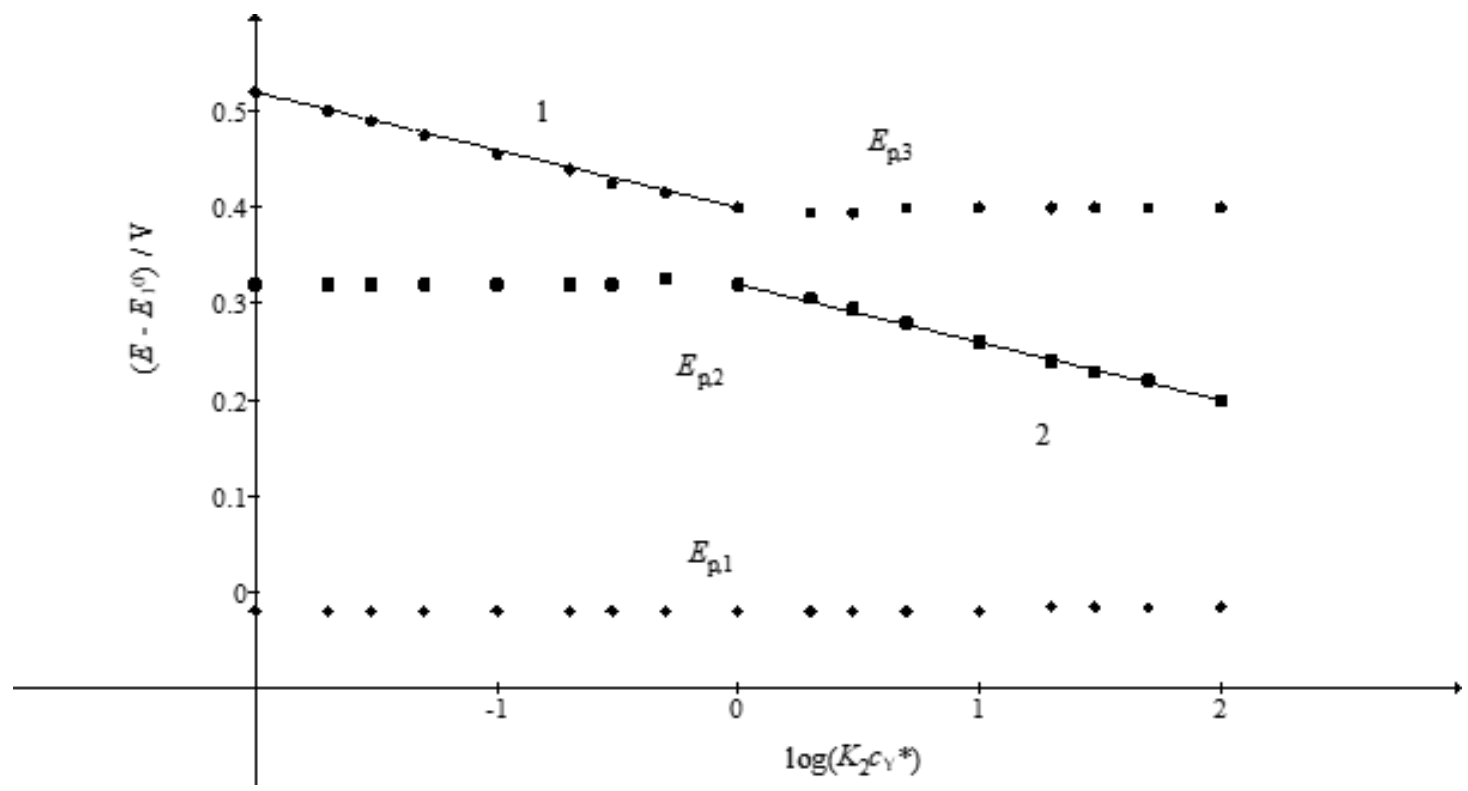

Fig. 6. Dependence of net peak potentials on the logarithm of dimensionless equilibrium constant of the second chemical reaction. $E_{2}^{0}-E_{1}^{0}=0.3 \mathrm{~V}, E_{3}^{0}-E_{1}^{0}=0.4 \mathrm{~V}$ and $K_{1} c_{X}^{0}=1$

The mixed response of the second and the third electrode reactions may split into two peaks if the second dimensionless equilibrium constant is bigger than the certain limiting value. Fig. 7 shows the relationship between the logarithm of this critical constant and the difference between the third and the second standard potentials. It satisfies the following equation:

$$
\log \left(K_{2} c_{\mathrm{Y}}^{*}\right)_{\text {crit. }}=-17.4\left(E_{3}^{0}-E_{2}^{0}\right)+2.176
$$

The last special case considers the possibility that the reagents $X^{-}$and $Y^{-}$are identical, so that their concentrations cannot be changed separately. In the simplest version of this mechanism it is assumed that $K_{1}=K_{2}$. Fig. 8 shows relationship between peak potentials and the logarithm of $K_{1} c_{\mathrm{X}}^{*}$ under this condition. It can be compared to Fig. 3. If $K_{1} c_{\mathrm{X}}^{*}<0.005$ the first peak corresponds to the single electron transfer, while the second peak is a consequence of the transfer of two electrons. On the contrary, if $K_{1} c_{\mathrm{X}}^{*}>500$ the first peak is twice as big as the second one. The slope of the straight line 1 is $-0.040 \mathrm{~V}$. This means that it satisfies eq. (42). Considering that $K_{2} c_{\mathrm{Y}}^{*}=K_{1} c_{\mathrm{X}}^{*}$ one obtains:

$$
E_{\mathrm{p}, 1}=\left(E_{1}^{0}+E_{2}^{0}+E_{3}^{0}\right) / 3-2.3(2 R T / 3 F) \log \left(K_{1} c_{\mathrm{X}}^{*}\right)
$$

The slopes of straight lines 2 and 3 are $-0.060 \mathrm{~V}$. They both describe mixed responses that include two electrons. The first one is the CECE mechanism and the second one is ECEC mechanism:

$$
\begin{aligned}
& E_{\mathrm{p}, 2}=\left[E_{2}^{0}-2(R T / F) \ln \left(K_{1} c_{\mathrm{X}}^{*}\right)+E_{3}^{0}\right] / 2 \\
& E_{\mathrm{p}, 2}=\left(E_{2}^{0}+E_{3}^{0}\right) / 2-2.3(R T / F) \log \left(K_{1} c_{\mathrm{X}}^{*}\right) \\
& E_{\mathrm{p}, 1}=\left(E_{1}^{0}+E_{2}^{0}\right) / 2-2.3(R T / F) \log \left(K_{1} c_{\mathrm{X}}^{*}\right)
\end{aligned}
$$




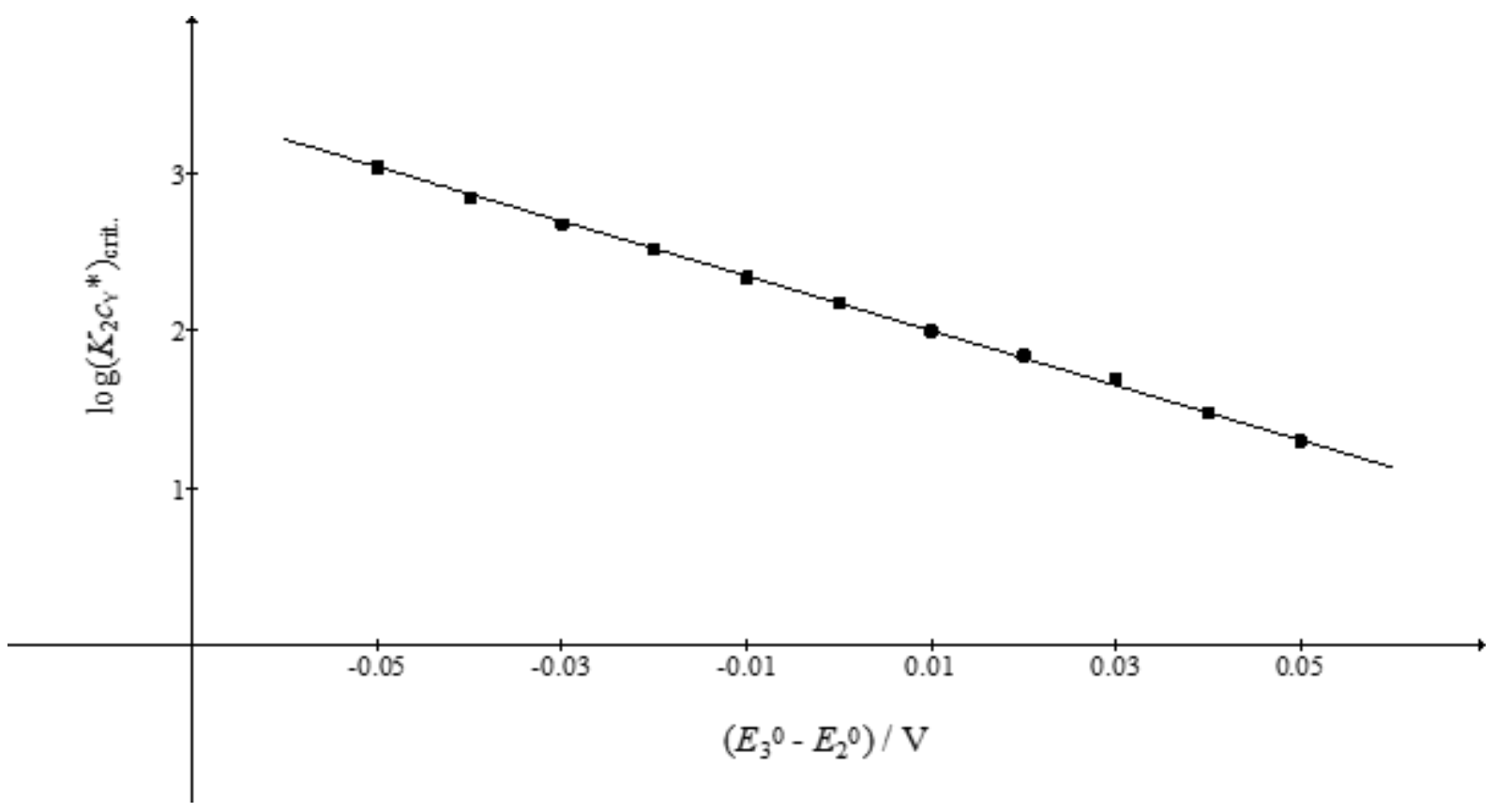

Fig. 7. Dependence of the critical value of the dimensionless equilibrium constant of the second chemical reaction on the difference between standard potentials of the third and the second electron transfer steps. $K_{1} c_{X}^{*}=1$ and $E_{2}^{0}-E_{1}^{0}=0.4 \mathrm{~V}$

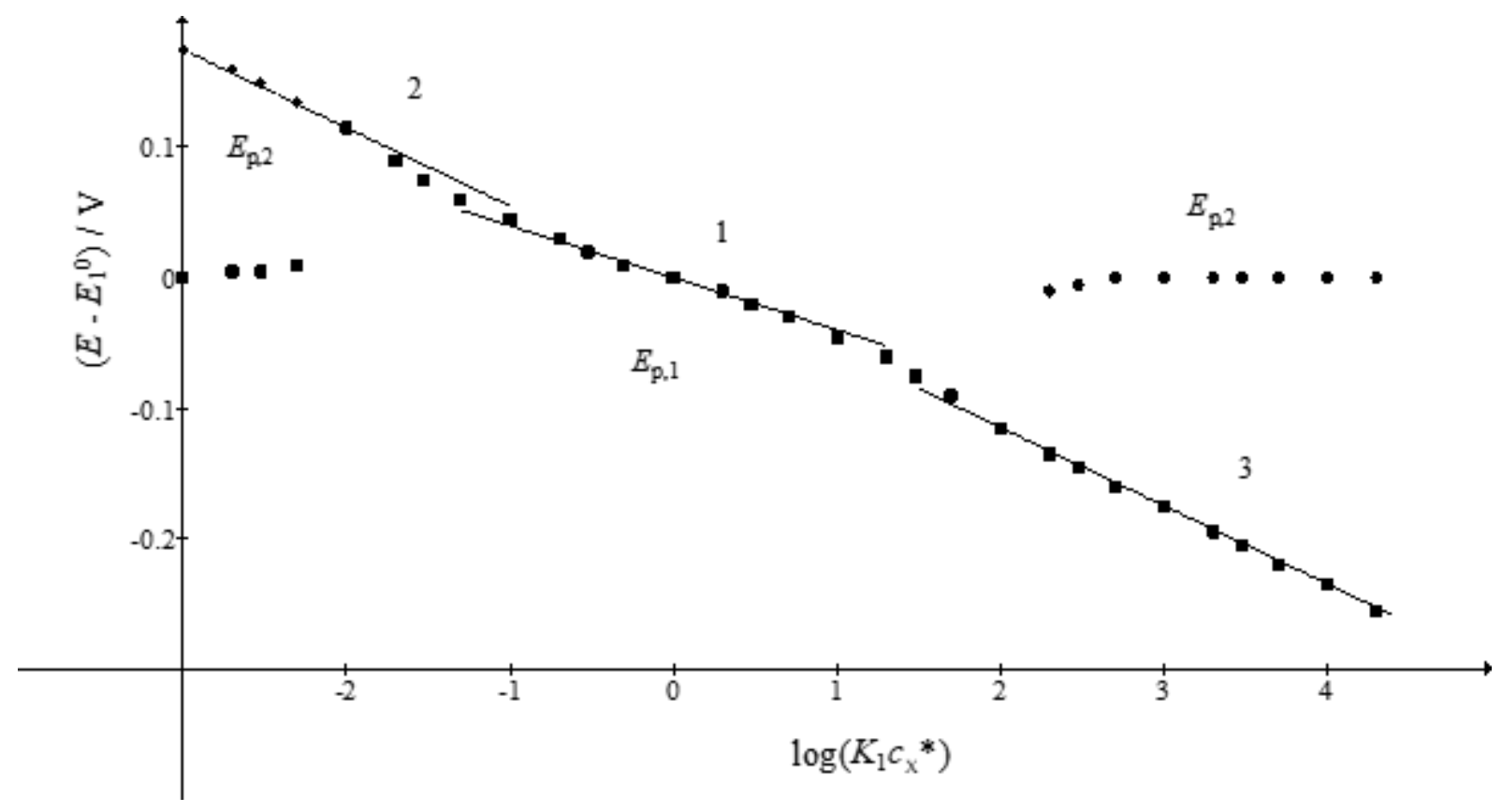

Fig. 8 Dependence of net peak potentials on the logarithm of the common dimensionless equilibrium constant. $E_{1}^{0}=E_{2}^{0}=E_{3}^{0}$ and $K_{2} c_{Y}^{*}=K_{1} c_{X}^{*}$

Comparing to Fig. 3, the response exhibits a single peak within rather narrow range $-1<\log \left(K_{1} c_{\mathrm{X}}^{*}\right)<1$,

\section{Conclusions}

The difference between the ECECE and the simple EEE mechanisms under equilibrium conditions is in the dependence of the former on the concentrations of the reagents of chemical reactions. By the variation of concentrations of these reagents, the potentials of peaks can be changed and the number of peaks can be increased under some conditions. Depending on standard potentials of electrode reactions, several special cases of ECECE mechanism can be distinguished. The first ECE 
mechanism can be analyzed separately if the third standard potential is much higher than the other two. Also, the second ECE mechanism appears isolated if the first standard potential is much lower than the other two. The influence of dimensionless equilibrium constants of chemical reactions on the certain peak potential depends on the number of electrons that are transferred at this potential. If chemical reactions include the same reagent, the first two, or the last two electrons may be transferred according to the ECEC, or CECE mechanisms, respectively.

\section{References}

[1] A. Molina, J. Gonzalez, E. Laborda, R. G. Compton, Int. J. Electrochem. Sci. 7 (2012) 5765 - 5778.

[2] A. Molina, E. Laborda, J. M. Gomez-Gil, R. G. Compton, J. Solid State Electrochem. 20 (2016) 3239 3253.

[3] M. Lovrić, Š. Komorsky-Lovrić, J. Electroanal. Chem. 735 (2014) 90 - 94.

[4] A. Molina, C. Serna, M. Lopez-Tenes, R. Chicon, Electrochem. Commun. 2 (2000) 267 - 271.

[5] R. Gulaboski, V. Markovski, Z. Jihe, J. Solid State Electrochem. 20 (2016) 3229 - 3238.

[6] C. Batchelor-McAuley, R. G. Compton, J. Electroanal. Chem. 669 (2012) 73 - 81.

[7] R. Gulaboski, P. Kokoškarova, S. Mitrev, Electrochim. Acta 69 (2012) 86 - 96.

[8] M. Lopez-Tenes, A. Molina, C. Serna, M. M. Moreno, J. Gonzalez, J. Electroanal. Chem. 603 (2007) 249 $-259$.

[9] A. Molina, C. Serna, M. Lopez-Tenes, M. M. Moreno, J. Electroanal. Chem. 576 (2005) 9 - 19.

[10] R. Gulaboski, V. Mirčeski, I. Bogeski, M. Hoth, J. Solid State Electrochem. 16 (2012) 2315 - 2328.

[11] S. O. Engblom, J. C. Myland, K. B. Oldham, Anal. Chem. 66 (1994) 3182 - 3187.

[12] A. B. Miles, R. G. Compton, J. Electroanal. Chem. 499 (2001) 1 - 16.

[13] J. J. O'Dea, K. Wikiel, J. Osteryoung, J. Phys. Chem. 94 (1990) 3628 - 3636.

[14] P. T. Sanecki, P. M. Skital, Electrochim. Acta 53 (2008) 7711 - 7719.

[15] Š. Komorsky-Lovrić, M. Lovrić, Anal. Bioanal. Electrochem. 5 (2013) 291 - 304.

[16] M. A. N. D. A. Lemos, A. J. L. Pombeiro, J. Organomet. Chem. 438 (1992) 159 - 165.

[17] J. D. Wadhawan, T. J. Davies, A. D. Clegg, N. S. Lawrence, J. C. Ball, O. V. Klymenko, N. V. Rees, D. Bethell, M. P. Woolfall, R. R. France, R. G. Compton, J. Electroanal. Chem. 533 (2002) 33 - 70.

[18] G. Kokkinidis, G. Papanastasiou, J. Electroanal. Chem. 257 (1988) 239 - 255.

[19] G. Papanastasiou, G. Kokkinidis, N. Papadopoulos, J. Electroanal. Chem. 305 (1991) 19 - 36.

[20] G. Kokkinidis, G. Papanastasiou, C. Hasiotis, N. Papadopoulos, J. Electroanal. Chem. 309 (1991) 263 272.

[21] D. Nematollahi, S. M. Golabi, J. Electroanal. Chem. 405 (1996) 133 - 140.

[22] D. Habibi, D. Nematollahi, Z. S. Al-Hoseini, S. Dehdashtian, Electrochim. Acta 52 (2006) 1234 - 1239.

[23] L. K. Bieniasz, Modelling Electroanalytical Experiments by the Integral Equation Method, Springer, Berlin, 2015.

[24] F. Scholz in: F. Scholz (Ed.), Electroanalytical Methods, Springer, Berlin, 2010, p. 11-31.

(C2017 by the authors; licensee IAPC, Zagreb, Croatia. This article is an open-access article distributed under the terms and conditions of the Creative Commons Attribution license (http://creativecommons.org/licenses/by/4.0/) 